\title{
Effect of Ketoprofenphonophoresis and Femoral Nerve Block in Knee Osteoarthritis
}

\author{
Fan Fan, Wei Wei, Binyan He, Chaonan Li, Qing Yang, Shuiqin Li* \\ Department of Rehabilitation Medicine, the First Affiliated Hospital of Xi'an Medical Universiy, Xi'an 710077, Shaanxi
} Province, China

*Corresponding author: Shuiqing Li, 1263304961@qq.com

\begin{abstract}
Objective: To evaluate the effect of ketoprofenphonophoresis and femoral nerve block in knee osteoarthritis. Methods: One hundred and fourteen patients with knee osteoarthritis were randomly divided into two groups. The control group consisted of 57 patients who were treated with only ketoprofenphonophoresis. The experimental group consisted of patients were treated with ketoprofenphonophoresis and femoral nerve block. The patients were followed for more than one month. The treatment effect assessed by observing their WOMAC index before and after therapy. Results: All the patients finished the follow-up. Their WOMAC indexes were reduced after therapy and one month after treatment, especially in the experimental group $(\mathrm{P}<0.05)$. The effective efficiency of ketoprofenphonophoresis and femoral nerve block were higher than that in the control group after treatment and after one month of follow-up $(\mathrm{P}<0.05)$. Conclusion: Compared with only ketoprofenphonophoresis, the combined treatment of ketoprofenphonophoresis and femoral nerve block was more appropriate in knee osteoarthritis.
\end{abstract}

Keywords: Ketoprofenphonophoresis; Femoral nerve block; Knee osteoarthritis

Publication date: November 2021; Online publication: November 30, 2021

\section{Introduction}

Knee osteoarthritis is a kind of retrograde degeneration of articular surface, which is mainly characterized by knee pain and limited activity that affect the quality of life of patients for a long time ${ }^{[1]}$. Its treatment methods include basic treatment, drug treatment, restorative treatment and reconstruction treatment. In addition to weight management, reasonable exercise and traditional Chinese medicine treatment, physical therapy is also one of the important treatment methods of basic treatment ${ }^{[2]}$. Among them, ultrasound therapy can improve the symptoms of knee arthritis, such as pain and limited joint activity ${ }^{[3]}$, and ketoprofen gel is often used as in drug introduction during treatment. Femoral nerve block also has a good analgesic effect on knee osteoarthritis ${ }^{[4]}$. This study aims to evaluate the effect of ketoprofenphonophoresis and femoral nerve block in knee osteoarthritis.

\section{Materials and methods}

\subsection{General information}

One hundred and fourteen patients with knee osteoarthritis treated in our hospital from December 2016 to December 2019 were randomly divided into two groups according to the random control table method. There were 57 cases in the ketoprofenphonophoresis group (the control group), including 26 males and 31 females, with an average age of $61.53 \pm 9.48$ years and an average course of $11.75 \pm 6.73$ months. There were 57 cases in the ketoprofenphonophoresis combined with femoral nerve block group (the experimental group), including 27 males and 30 females, with an average age of $60.19 \pm 9.78$ years and an average course 
of 10.22 \pm 5.56 months. There was no significant difference in gender, age, course of disease and WOMAC score between the two groups (Table 1 and Table 2).

Table 1. Patients' information in two groups

\begin{tabular}{lccccc}
\hline \multirow{2}{*}{ Group } & \multicolumn{2}{c}{ Number of cases } & & Average age (years) & Course of disease (months) \\
\cline { 2 - 3 } Control group & Male & Female & & $61.53 \pm 9.48$ & $11.75 \pm 6.73$ \\
Experimental group & 26 & 31 & & $60.19 \pm 9.78$ & $10.22 \pm 5.56$ \\
\hline
\end{tabular}

Table 2. WOMAC index before and after therapy

\begin{tabular}{lccc} 
Group & Pre-treatment score & Post-treatment score & Scores scored 1 month after treatment \\
\hline Control group & $85.52 \pm 9.49$ & $39.05 \pm 18.75^{*}$ & $39.88 \pm 19.33^{*}$ \\
Experimental group & $81.86 \pm 12.49$ & $31.44 \pm 17.04^{* \#}$ & $32.82 \pm 19.13^{* \#}$ \\
$\mathrm{Z}$ & -1.466 & -2.461 & -2.205 \\
$\mathrm{P}$ & 0.143 & 0.014 & 0.027 \\
\hline
\end{tabular}

$* \mathrm{P}<0.05$, compared with before therapy; ${ }^{\#} \mathrm{P}<0.05$, compared with the control group

\subsection{Inclusion and exclusion criteria}

The inclusion criteria are as follows: (1) patient meeting the diagnostic criteria of knee osteoarthritis ${ }^{[5]}$; (2) patient aged > 50 years; (3) no treatment received for more than 2 months; (3) patient willing to receive treatment and sign informed consent.

The exclusion criteria are as follows: (1) patient unwilling to accept the above-mentioned treatment; (2) patient who suffers from systemic diseases leading to osteoarthritis; (3) patient who are taking or receiving other treatments recently, or who have recently used hormones; (4) patient with tumor, tuberculosis, hematopathy, psychosis, hyperthyroidism and hyperparathyroidism, as well as pregnant and lactating women; (5) patient with severe heart, lung, liver and kidney diseases, diabetes, and bleeding tendency.

\subsection{Treatment method}

All 57 patients of the control group were treated with ketoprofenphonophoresis therapy. The US-750 ultrasonic therapy apparatus (Ito Physiotherapy \& Rehabilitation Co., Ltd.) was used. The iontophoresis process was introduced into the OTM mode. The diameter of the head was $2.5 \mathrm{~cm}$, the output of frequency was $3 \mathrm{MHz}$, the sound intensity dose was $0.75-1.25 \mathrm{~W} / \mathrm{cm}^{2}$. The Ketoprofen Gel was used in the treatment of the acoustic head and the knee joint (the product name is "Remai," manufactured by Hanmei Pharm. Co., Ltd., and packaged by Beijing Hanmi Pharm. Co., Ltd.). The method of movement was adopted, taking the suprapatellar sac, the medial and lateral knee eyes and the local pain of the knee as the center, with 15 min each time, once a day, and 2 weeks as a course of treatment.

In the experimental group, 57 patients were treated with ketoprofenphonophoresis combined with femoral nerve block, and the treatment method of ketoprofenphonophoresis was the same as that of the 
control group. For femoral nerve block, $5 \mathrm{ml}$ of $2 \%$ lidocaine hydrochloride injection and $0.5 \mathrm{mg}$ of vitamin B12 injection were mixed, $20 \mathrm{mg}$ of triamcinolone acetonide acetate injection was added once a week, and $10 \mathrm{ml}$ with $0.9 \%$ sodium chloride injection was diluted. The patient took the supine position; the lower limbs were slightly apart, the affected foot was rotated outwards, and the femoral artery can be found by palpation about $1-2 \mathrm{~cm}$ below the midpoint of the inguinal ligament. The lateral $2 \mathrm{~cm}$ is the insertion point. We can hold the No.7 4-5cm puncture needle vertically and slowly insert the needle. The insertion of needle was stopped when there is abnormal sensation. The needle was rotated $360^{\circ}$, and the drug was injected after suction in the absence of blood. The drug should be taken three times a week, and a course of treatment took two weeks.

\subsection{Observation indexes}

The process of WOMAC (the Western Ontario and McMaster Universities Osteoarthritis Index) was scored by $10 \mathrm{~cm}$ visual analogue scale (VAS) before, after, and 1 month after treatment. The WOMAC includes 5 pain scores, 2 stiffness scores, and 17 joint function scores; there are 24 items total, with 10 points for each item and 240 points in total. The total score and its difference before and after treatment were calculated. The curative effect is determined according to the percentage of difference reduction, and the formula is as follows:

$$
\text { Curative effect }=(\text { before treatment }- \text { after treatment }) / \text { before treatment } \times 100 \%
$$

The efficacy criteria are as follows: Invalid $\leq 25 \% ; 25 \%<$ effective $\leq 50 \% ; 50 \%<\operatorname{good} \leq 75 \% ; 75 \%$ $<$ clinically cured $\leq 100 \%{ }^{[6]}$.

\subsection{Follow-up}

The follow-up started after the treatment and lasted for 1 month. The patients were followed up every 2 weeks, and the changes of WOMAC score were followed up by outpatient follow-up and visit.

\subsection{Statistical methods}

SPSS 25.0 software was used for statistical analysis, in which WOMAC score was expressed as mean \pm standard deviation (SD). The scores of the two groups before and after treatment were measured, and the curative effect was counted. The Mann-Whitney test in the rank sum test was used for the comparison between the two groups. If $\mathrm{P}<0.05$, it means that the difference was statistically significant.

\section{Results}

\subsection{Follow-up}

One hundred and fourteen patients with knee osteoarthritis were followed up for more than 1 month. All patients completed the follow-up, and the distribution characteristics of population data were similar.

\subsection{Comparison of WOMAC scores between the two groups before and after treatment}

There was no significant difference in WOMAC score of 114 patients before treatment $(\mathrm{P}>0.05)$. After treatment, the scores of the two groups were lower than those before treatment: control group is $85.52 \pm 9.49$ vs $39.05 \pm 18.75$, and the experimental group is $81.86 \pm 12.49$ vs $31.44 \pm 17.04$. The decrease was more pronounced in the research group $(\mathrm{Z}=-2.461, \mathrm{P}<0.05)$. One month after treatment, the WOMAC score of the two groups was still lower than that before treatment: control group is $85.52 \pm 9.49$ vs $39.88 \pm 19.33$, and the experimental group is $81.86 \pm 12.49$ vs $32.82 \pm 19.13$. The decrease was more apparent in the experimental group, and the difference was statistically significant $(Z=-2.205, P<0.05)$, as shown in Table 2. 


\subsection{Comparison of curative effects between the two groups after treatment}

The percentage of WOMAC score difference between the two groups before and after treatment was calculated. After treatment, the invalid, effective, good and cured patients in each group were: 5, 14, 28 and 10 patients in the control group, and 2, 8, 29 and 18 patients in the experimental group. The total effective rate of the latter was higher (91.22\% vs $96.49 \%)$, and the difference was statistically significant $(\mathrm{Z}=-2.268$, $\mathrm{P}<0.05$ ), as shown in Table 3. One month after treatment, the ineffective, effective, good and cured patients in each group were: $6,15,26$ and 10 patients in the control group, and 2, 11, 26 and 18 patients in the experimental group. The total effective rate of the latter was higher $(89.47 \%$ vs $96.49 \%)$, and the difference was statistically significant $(Z=-2.117, \mathrm{P}<0.05)$, as shown in Table 4.

Table 3. Effective efficiency after therapy

\begin{tabular}{|c|c|c|c|c|c|c|c|}
\hline \multirow{2}{*}{ Group } & \multicolumn{4}{|c|}{ Number of cases with curative effect } & \multirow{2}{*}{$\begin{array}{c}\text { Total effective } \\
\text { rate }\end{array}$} & \multirow{2}{*}{$\mathbf{Z}$} & \multirow{2}{*}{$\mathbf{P}$} \\
\hline & Inefficient & Effective & Good & Cured & & & \\
\hline Control group & 5 & 14 & 28 & 10 & $91.22 \%$ & \multirow{2}{*}{-2.268} & \multirow{2}{*}{0.023} \\
\hline Research group & 2 & 8 & 29 & 18 & $96.49 \%$ & & \\
\hline
\end{tabular}

Table 4. Effective efficiency one month after therapy

\begin{tabular}{|c|c|c|c|c|c|c|c|}
\hline \multirow{2}{*}{ Group } & \multicolumn{4}{|c|}{ Number of cases with curative effect } & \multirow{2}{*}{$\begin{array}{c}\text { Total effective } \\
\text { rate }\end{array}$} & \multirow{2}{*}{$\mathbf{Z}$} & \multirow{2}{*}{$\mathbf{P}$} \\
\hline & Inefficient & Effective & Good & Cured & & & \\
\hline Control group & 6 & 15 & 26 & 10 & $89.47 \%$ & \multirow{2}{*}{-2.117} & \multirow{2}{*}{0.034} \\
\hline Experimental group & 2 & 11 & 26 & 18 & $96.49 \%$ & & \\
\hline
\end{tabular}

\section{Discussion}

At present, 250 million people worldwide suffer from knee osteoarthritis, and most individuals with the symptoms are aged over 50 years old ${ }^{[7]}$. Although knee osteoarthritis has no obvious lethality and the disability rate is lower than that of rheumatism arthritis or rheumatoid arthritis, it has the greatest impact on the quality of life of the elderly because of its high prevalence. Age, gender, weight, malnutrition, cold environment, joint injury, osteoporosis and other factors are related to its occurrence, but its pathogenesis is still unclear ${ }^{[8]}$.

It is found that the main sign of osteoarthritis is cartilage degeneration. The imbalance between the synthesis and degradation of articular cartilage extracellular matrix (ECM) is one of the important reasons for cartilage degeneration. Matrix metalloproteinase (MMP) may play a decisive role. This substance is also one of the main basic factors for physiological reconstruction and pathological destruction ${ }^{[9]}$. In the process of osteoarthritis, articular chondrocytes suffer a series of damages, including abnormal biomechanical stress and influence from pro-inflammatory cytokines and chemokines, which will lead to the phenotypic transfer of chondrocytes and the aggravation of the lesions ${ }^{[10]}$. Therefore, delaying cartilage degeneration, improving biomechanical balance and eliminating inflammatory reaction are some of the proposed ideas in the treatment of knee arthritis. It is important to note that an earlier intervention is conducive to the prognosis.

Results from animal experiments show that ultrasound can inhibit the expression of MMP-1 and MMP-13 in rabbit knee osteoarthritis cartilage, so as to reduce the destructive effect of MMP and promote 
chondrocyte repair ${ }^{[11]}$. Previously, a meta-analysis summarized the safety and effectiveness of ultrasonic treatment in 1074 patients with knee osteoarthritis. The results suggest that ultrasonic treatment can significantly reduce knee pain and increase joint mobility ${ }^{[12]}$. Ketoprofen Gel, as an excellent 2- aryl propionic acid nonsteroidal anti-inflammatory analgesic drug, can inhibit the biological activity of cyclooxygenase (COXs) and lipoxygenase (LOXs) in vivo, so as to inhibit the synthesis of prostaglandin (PGs) and leukotriene (LTs) ${ }^{[13]}$. The external administration route can not only alleviate the pain, swelling, tenderness and other uncomfortable symptoms, but also reduce the gastrointestinal irritation caused by the oral drugs. The fat emulsion in the water-based gel can act as an ultrasonic coupling agent to promote drug absorption through cavitation effect. Clinical observation confirmed that ketoprofenphonophoresis is more effective than ultrasound in the treatment of elderly knee osteoarthritis ${ }^{[14]}$. However, both simple ultrasound therapy and ketoprofenphonophoresis therapy have a slow treatment effect, which warrants a longer time (ranging from 4 to 8 weeks) for continuous treatment ${ }^{[15]}$.

As one of the important methods of surgical anesthesia, nerve block is also used in the analgesic treatment of clinical diseases. This method does not puncture around the joint in the treatment of knee osteoarthritis, does not damage the joint capsule, and has no adverse effect on gastrointestinal function. Studies have confirmed that nerve block can inhibit the release of substance $\mathrm{P}$, promote blood circulation in innervated areas, promote inflammatory absorption, and accelerate tissue damage repair ${ }^{[16]}$. Nerve block on lumbosacral plexus and its branches (lumbosacral 2-sacral 1, i.e., femoral nerve, lateral femoral cutaneous nerve and sciatic nerve) can block the mechanical imbalance of knee joint and significantly reduce the contents of interleukin- $1 \beta$, tumor necrosis factor- $\alpha$ (TNF- $\alpha$ ), nitric oxide (NO) and malondialdehyde in knee joint fluid ${ }^{[17]}$. The femoral nerve block belongs to unilateral block, without sympathetic nerve block and urinary retention symptom, and has higher accuracy than other lumbosacral plexus nerve blocks. Clinical data show that femoral nerve block in patients with knee osteoarthritis can better improve the symptoms of pain, swelling and joint stiffness than psoas intermuscular sulci nerve block [18].

In this study, ketoprofenphonophoresis therapy was combined with femoral nerve block therapy. The results showed that the combination of the two can significantly reduce the WOMAC score of patients with knee osteoarthritis, and the total effective rate is significantly higher than that of the simple ketoprofenphonophoresis treatment group $(\mathrm{P}<0.05)$. Most patients with knee arthritis (especially those in the acute inflammatory stage) can significantly alleviate the symptoms of knee pain, swelling and joint stiffness in a short time after combined treatment is used. Nevertheless, this form of treatment can only alleviate some symptoms of pain and swelling in patients of chronic, middle and late stage and over 75 years old, with no significant improvement in joint stiffness and deformation.

Compared with the 4-8 weeks of ultrasound treatment with ketoprofen alone, the time of femoral nerve block combined with ketoprofen ultrasound treatment in this research took only 2 weeks and demonstrated rapid and stable effect. There is no obvious sign of relapse one month after treatment. The reason is that femoral nerve block can inhibit substance $\mathrm{P}$ and reduce TNF- $\alpha$ and inflammatory factors such as NO, as well as improve knee inflammation and mechanical imbalance. Ketoprofenphonophoresis therapy can reduce inflammation and swelling as well as promote cartilage repair. The combined treatment of the two can quickly alleviate the internal inflammatory reaction of the knee joint, so as to delay the cartilage degeneration injury and break the vicious circle of inflammation and cartilage degeneration. At present, this study only analyzes and compares the short-term efficacy process of patients after treatment. The longterm prognosis of the two groups still needs to be further analyzed and confirmed. From an economic point of view, femoral nerve block and ketoprofenphonophoresis therapy are less costly and easy to operate as well as have little side effects. They can significantly improve the discomfort symptoms and quality of life of patients with knee osteoarthritis in the short term. The combined treatment of the two can be used as one 
of the important conservative treatment methods for patients with knee osteoarthritis.

According to the step-by-step treatment scheme of knee osteoarthritis, basic treatment should be adopted in the initial, early, middle and late stages of knee osteoarthritis. Prevention is the main focus in the initial and early stages, and auxiliary restorative treatment and surgical treatment are the main focus in the middle and late stages. Although the application of nerve block in the treatment of knee osteoarthritis has not been mentioned in the updated relevant guidelines ${ }^{[19]}$, a growing number of studies have confirmed its effectiveness and safety. The combination of femoral nerve block and ketoprofenphonophoresis therapy can benefit patients with knee osteoarthritis.

\section{Disclosure statement}

The authors declare no conflict of interest.

\section{References}

[1] Tang X, Wang SF, Zhan SY, et al., 2016, The Prevalence of Symptomatic Knee Osteoarthritis in China Results from the China Health and Retirement Longitudinal Study. Arthritis Rheumatol, 68(3): 648653.

[2] Joint Surgery Group, 2019, Orthopaedic Branch, Chinese Medical Association Expert Consensus on Step Therapy for Knee Osteoarthritis (2018 Edition). Chinese Journal of Joint Surgery (Electronic Edition), 13(1): 124-130.

[3] Wu H, Deng HW, Liu LP, et al., 2012, Clinical Study of Focused Ultrasound in Treatment of Knee Osteoarthritis. Chinese Journal of Postgraduates of Medicine, 35(8): 39-41.

[4] Zhang LN, 2016, Clinical Observation of Ultrasound-guided Femoral Nerve Block. China Practical Medicine, 11(24): 22-23.

[5] Chinese Medical Association, 2013, Clinical Technical Operation Specification: Pain Volume.People's Military Medical Press, Beijing, 78-87.

[6] Chinese Medical Association, 2007, Clinical Diagnosis and Treatment Guidelines: Pain volume, People's Medical Publishing House, Beijing, 14-15.

[7] Vos T, Flaxman AD, Naghavi M, et al., 2012, Years Lived with Disability (YLDs) for 1160 Sequelae of 289 Diseases and Injuries 1990-2010: A Systematic Analysis for the Global Burden of Disease Study 2010. Lancet, 380(9859): 63-96.

[8] Wu ZJ, 2018, Etiology, Pathogenesis and Treatment Progress of Knee Osteoarthritis. Chinese Journal of Medical Device, 31(4): 203-204.

[9] Khan M, Adili A, Winemaker M, et al., 2018, Management of Osteoarthritis of the Knee in Patients. CMAJ, 190(3): E72-E79.

[10] Zheng XF, 2017, Pathogenesis and Recent Advances in Treatment of Osteoarthritis. Chinese Journal of Tissue Engineering Research, 21(20): 3255-3262.

[11] Li WP, Jian R, Xu FY, 2012, Effect of Ultrasonic Treatment on the Expression of MMP-1 and MMP13 in Rabbit Knee Osteoarthritis. Chongqing Medical, 41(16): 1564-1566.

[12] Wu Y, Zhu S, Lv Z, et al., 2019, Effects of Therapeutic Ultrasound for Knee Osteoarthritis: A Systematic Review and Meta-Analysis. SAGE Journals, 33(12):1863-1875.

[13] Jain M, Srivastava V, Kumar R, et al., 2017, Determination of Five Potential Genotoxic Impurities in Dalfampridine Using Liquid Chromatography. J Pharm Biomed Anal, 133(3): 27-31. 
[14] Guan S, Duan XQ, Qiao SX, et al., 2010, Clinical Observation of Ketoprofenphonophoresis In the Treatment of Senile Knee Osteoarthritis. Chinese Journal of Gerontology, 3(30): 582-583.

[15] Wang YD, 2018, Effect of Ultrasound Combined with Sodium Hyaluronate on Knee Function in Patients with Knee Osteoarthritis. Journal of Frontier of Medicine, 8(28): 122-123.

[16] Fan GM, Gong SL, Dai JP, et al., 2012, Effect of Femoral Nerve Block on Serum Substance P Content in Rabbits with Osteoarthritis. Chinese Journal of Pain Medicine, 18(4): 238-241.

[17] Wang DS, Wang L, Chen Q, et al., 2010, Effect of Lumbosacral Plexus and Branch Nerve Block on Cytokine Content in Articular Fluid of Knee Osteoarthritis. Chinese Journal of Painology, 6(41): 266268.

[18] Lei L, Wang L, Wei W, 2017, Comparative Study of Two Nerve Block Therapies in the Treatment of Knee Osteoarthritis. Chinese Journal of Pain Medicine, 23(4): 307-309.

[19] Bannuru RR, Osani MC, Vaysbrot EE, et al., 2019, OARSI Guidelines for the Non-Surgical Management of Knee, Hip, and Polyarticularosteoarthritis. Osteoarthritis and Cartilage, 6(11): 1-35. 